\title{
A Composite Deep Learning Access for Leaf Species Classification
}

\author{
Yashvardhan Singh Rautela, Kanu Garg, Harshit Singh Chhabra, Rahul Nijhawan
}

\begin{abstract}
Plants are an integral part of the human life one way or the other. They have multi-dimensional use as food, medicine, clothing, art, industrial raw material and are vital for sustaining the ecological balance of our planet. All these real life applications make the identification of plants intensely important and useful. This dictates to design an accurate recognition system of plants. It will be useful to facilitate faster classification, management and apprehension. Almost all the plants are accompanied by unique leaves. In this paper, we have used this property of leaf identification for the identification of plants. In this study, we have applied a composite deep learning model, where Inception-v3 model is used for feature engineering and Stacking Ensemble model is used for the detection and classification of leaves from images. We have used a modified Flavia dataset of 1287 leaf images divided amongst 21 distinct plant species to test the proposed approach. On comparing our proposed work with other pre-existing algorithms (RF, SVM, $k N N$ and Tree), it is found that it surpassed them, obtaining an accuracy of $99.5 \%$.
\end{abstract}

Keywords: Deep Learning, Leaf Classification, Hybrid, Feature Extraction.

\section{INTRODUCTION}

Most of the plants have leaves in some form or other to sustain their life. In a vascular plant, leaves are specialized for photosynthesis. Plants can be identified solely on the basis of leaves. Leaves of certain plants have medicinal properties and are therefore used in the field of medicine and Ayurveda [1] [2]. Leaves of plants like tobacco and marijuana are used for commercial drug production. Leaves from hemp, henequen, sisal and abaca are used in the manufacturing of fibers [3]. Certain plants produce leaves that are a source of dyes and colors, like henna [4]. Leaves from banana, palm and areca trees are used as eco-friendly alternative to single-use utensils.

Cinnamon has multiple health benefits ranging from having anti-diabetic effect [5] to having antioxidants [6]. Deodar oil is useful in treating arthritis and headache. Sweet osmanthus is used in traditional Chinese medicine to treat irregular menstruation [7]. Along its traditional uses, camphor is used in the treatment of mania [8]. Ginkgo is used in the treatment of dementia and cognitive impairment [9].

Revised Manuscript Received on September 10, 2019.

Yashvardhan Singh Rautela, Graphic Era University, Dehradun (U.K.) India

(Email: yashvardhanrautela@gmail.com)

Kanu Garg, Graphic Era University, Dehradun (U.K.) India

(Email: kanugargng@gmail.com)

Harshit Singh Chhabra, Graphic Era University, Dehradun (U.K.) India

(Email: harshitchhabra8@gmail.com)

Rahul Nijhawan, Graphic Era University, Dehradun (U.K.) India

(Email: dr.rahul.iitr@gmail.com)
The field of classification of leaf species has an enormous potential for research. A considerable amount of progress has been made on plant classification and recognition on the basis of the shape of the leaves. Yigit et al. [10] have used Support vector machine (SVM), Random Forest algorithm, Naive Bayes algorithm, Artificial Neural

Network and k-Nearest Neighbor $(\mathrm{kNN})$ on 637 images of 32 varieties and obtained accuracy of $92.91 \%$. Sharma et al. [11] have used Artificial Neural Network on 1900 images of 18 species to obtain an accuracy of $97 \%$. Aliyu et al. [12] used Wiener filtering technique (WFT) on a dataset of 400 leaves from ten different species and obtained an accuracy of $95.1 \%$. Jalal et al. [13] used saliency detection and multilevel classification on a dataset with 13 species with 25 images each and obtained an accuracy of 94\%. Du et al. [14] have used MMC (Move Median Centers) classifier on a dataset of 400 images, divided amongst 20 species to obtain an accuracy of $91 \%$. Hossain et al. [15] extracted a binary image of the leaves, then extracted several morphological features from it. An Artificial Neural Network was trained on these features to classify leaves into 30 species. They obtained an accuracy of $91.41 \%$. Rankothge et al. [17] used Advanced Plant Identification System (APIS) to obtain an accuracy of $95 \%$.

The biggest challenge is to generate a big and diverse dataset. Finding such a dataset was a laborious process. Another challenge faced was the similarity in different species of leaves. Leaf classification was strenuous due to large number of almost identical features.

In our paper a composite deep learning model for leaf classification is proposed. We employed pre-trained Inception-v3 model [18], which is a variation of GoogLeNet [19], to get the feature vector of the images. Then we used a combination of different stacking models to get the results. It was observed that the anticipated model outperformed the contemporary algorithms. Further accuracy assessment has been performed using statistical measures (Accuracy, Specificity, Sensitivity, F1 score and AUC).

This paper is divided in the following order: I. Introduction, II. Data Collection, III. Methodology, IV. Proposed approach, V. Results and Discussions, VI. Conclusion.

\section{DATA COLLECTION}

Flavia dataset with digital images of different leaf species was used in our study for leaf classification [25]. We used 21 different species of leaves with 1287 images from this dataset. 
TABLE I

DISTRIBUTION OF IMAGES IN THE DATASET

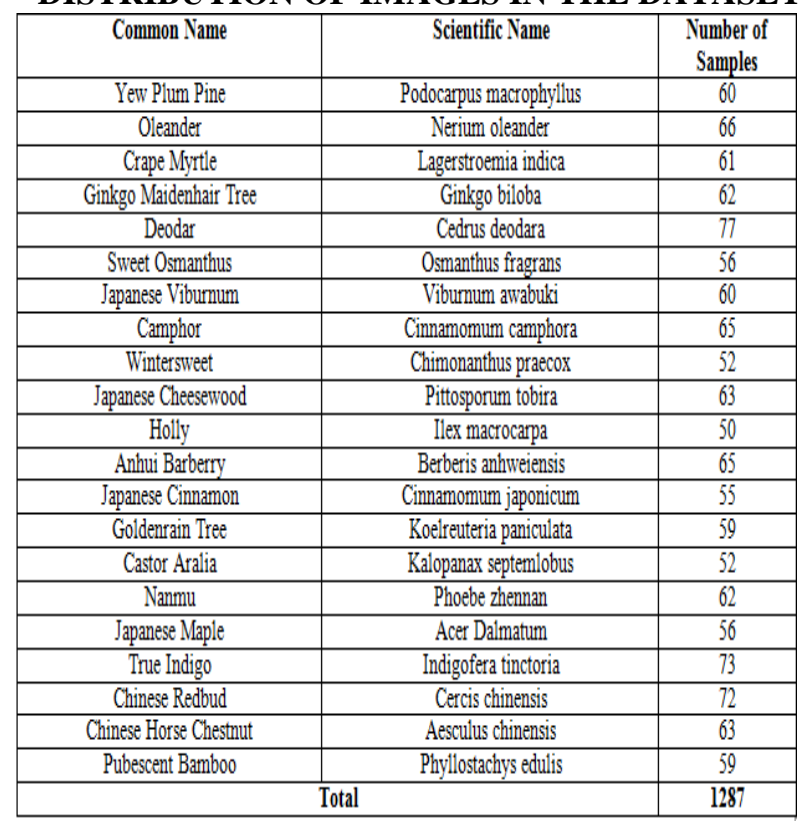

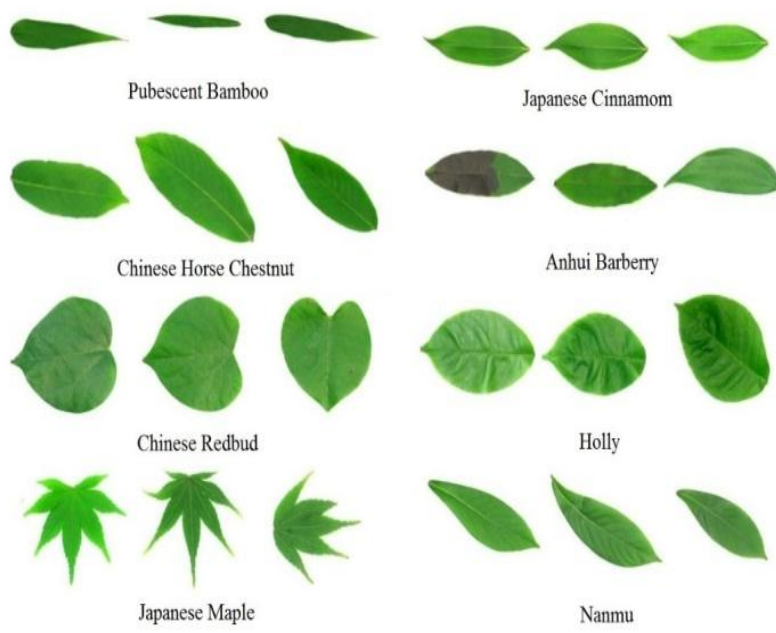

Figure 1. Sample of different types of leaves in our dataset

\section{METHODOLOGY}

In this paper, we used Ensemble modeling to perform classification of our dataset. We carried out our research on a machine having octa-core Intel I5 processor with $8 \mathrm{~GB}$ RAM and 2 GB NVidia GPU.

We performed feature extraction using pre-trained Inception-v3 model to get the features of images in our dataset. We also implemented our dataset on some contemporary algorithms like SVM, kNN, AdaBoost and Random Forest. Using statistical measures, we compared the results with our proposed approach.

\section{PROPOSED APPROACH}

In this paper we have used Inception-v3 model for feature extraction from the dataset. After that these features are applied on various stacking models for classifying the dataset.

Inception-v3 [17] [18] is a widely used model which was heavily engineered to improve speed and accuracy. A basic
Inception module has convolution filters of size $1 * 1,3 * 3$ and $5 * 5$ and pooling layer. 9 such modules were combined linearly and GoogLeNet (Inception-v1) was made. GoogLeNet model is 27 layers deep (max-pooling included). In the next version (Inception-v2), bigger filters $(5 * 5$ and $3 * 3)$ were broken into smaller $1 * 1$ filters to increase computation speed. The v3 model incorporates all the features of previous versions of Inception model. It also factorized the $7 * 7$ convolutions and applied label smoothing which helped to prevent over fitting.

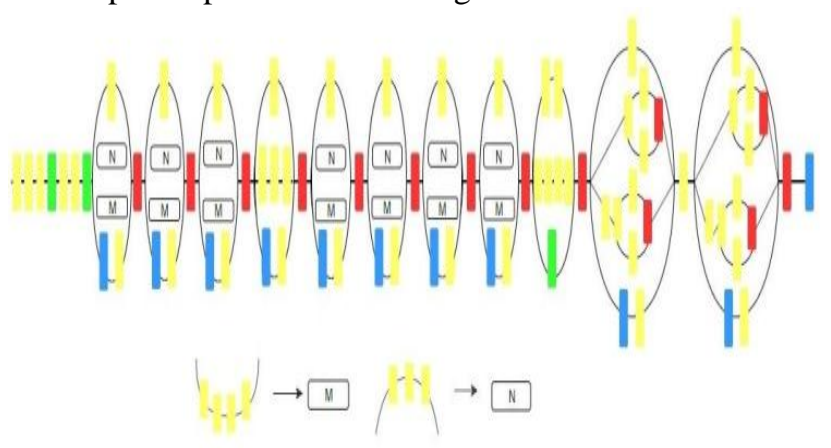

Figure 2. Feature Extraction: Inception-v3 model

Then we used an ensemble model to classify leaves from our dataset. Stacking is an Ensemble model which is used for pattern recognition and classification. It combines the predictions of two or more models (base-classifiers) on the same dataset and combines them using a meta-classifier to make a new model. Our proposed work uses Support Vector Machine, Logistic Regression and Artificial Neural Network as base classifiers. The predictions of the base classifiers were combined using Logistic Regression to obtain the final result.

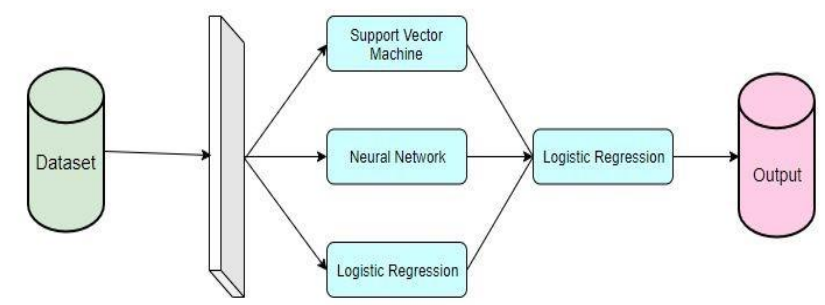

$$
\begin{gathered}
\text { Feature } \\
\text { Extraction }
\end{gathered}
$$

Figure 3. Proposed Model for Leaf Classification

\section{RESULTS AND DISCUSSION}

We have used 3 different pre-trained models (Inceptionv3, VGG16 and VGG19) for feature extraction of dataset. Then we have applied various contemporary algorithms and compared the results with different stacking models. Each stacking model was built with different. The various stacking approaches were:

Published By: 


\section{A. Stacking Approach 1:}

In this approach, we used Artificial Neural Network, Logistic Regression and Support Vector Machine [21] as base classifiers. Results were combined by Logistic Regression. We achieved an accuracy of $99.5 \%$ when Inception-v3 model was used.

\section{B. $\quad$ Stacking Approach 2:}

In this approach, we used AdaBoost [22], Support Vector Machine (kernel - RBF) and kNN [23] as base classifiers. Results were combined by Logistic Regression. We achieved an accuracy of $98.4 \%$ when VGG19 model was used.

\section{Stacking Approach 3:}

In this approach, we used AdaBoost, Random Forest [24] and $\mathrm{kNN}$ as base classifiers. Results were combined by Logistic Regression. We achieved an accuracy of $98.4 \%$ when VGG19 model was used.

\section{TABLE II}

\section{ACCURACY ASSESSMENT}

\begin{tabular}{|c|c|c|c|c|c|c|}
\hline $\begin{array}{c}\text { Feature } \\
\text { Extraction }\end{array}$ & Classifier & Accuracy & F1 Score & Precision & Sensitivity & AUC \\
\hline \multirow{5}{*}{ Inception v3 } & Stack 1 & 99.5 & 99.5 & 99.5 & 99.5 & 100 \\
\cline { 2 - 7 } & Stack 2 & 97.4 & 97.4 & 97.7 & 97.4 & 100 \\
\cline { 2 - 7 } & Stack 3 & 96.8 & 96.9 & 97.2 & 96.8 & 99.9 \\
\cline { 2 - 7 } & $\mathrm{kNN}$ & 96.3 & 96.3 & 96.6 & 96.3 & 99.7 \\
\cline { 2 - 7 } & $\mathrm{RF}$ & 92.6 & 92.5 & 93.2 & 92.6 & 99.8 \\
\cline { 2 - 7 } & AdaBoost & 80.4 & 80.6 & 82.9 & 80.4 & 89.7 \\
\hline \multirow{5}{*}{ VGG-16 } & Stack 1 & 98.9 & 98.9 & 99.1 & 98.9 & 100 \\
\cline { 2 - 7 } & Stack 2 & 97.9 & 97.9 & 98.3 & 97.9 & 100 \\
\cline { 2 - 7 } & $\mathrm{RF}$ & 96.8 & 96.8 & 97.2 & 96.8 & 99.8 \\
\cline { 2 - 7 } & Stack 3 & 96.8 & 96.6 & 97.3 & 96.8 & 99.9 \\
\cline { 2 - 7 } & $\mathrm{kNN}$ & 95.8 & 95.1 & 95.0 & 95.8 & 99.9 \\
\cline { 2 - 7 } & AdaBoost & 87.8 & 88.3 & 90.2 & 87.8 & 93.6 \\
\hline \multirow{5}{*}{ VGG-19 } & Stack 1 & 98.9 & 98.9 & 99.0 & 98.9 & 100 \\
\cline { 2 - 7 } & $\mathrm{kNN}$ & 98.4 & 98.4 & 98.5 & 98.4 & 99.7 \\
\cline { 2 - 7 } & Stack 2 & 98.4 & 98.4 & 98.5 & 98.4 & 100 \\
\cline { 2 - 7 } & Stack 3 & 98.4 & 98.4 & 98.4 & 98.4 & 100 \\
\cline { 2 - 6 } & $\mathrm{RF}$ & 94.7 & 94.8 & 95.4 & 94.7 & 99.9 \\
\cline { 2 - 6 } & AdaBoost & 79.9 & 79.8 & 82.2 & 79.9 & 89.5 \\
\hline
\end{tabular}

Our proposed approach (Stacking Approach 1) gave best results as shown in Table II. We achieved a classification accuracy of $99.5 \%$ with precision $99.5 \%$, F1 Score $99.5 \%$ and Sensitivity $99.5 \%$. By using ROC curve shown in Fig. 4 the performance of this model has been estimated. ROC curve is built by using TP Rate on the y-axis and FP Rate on the $\mathrm{X}$-axis. If the value of ROC is close to 1 then a model is considered perfect.

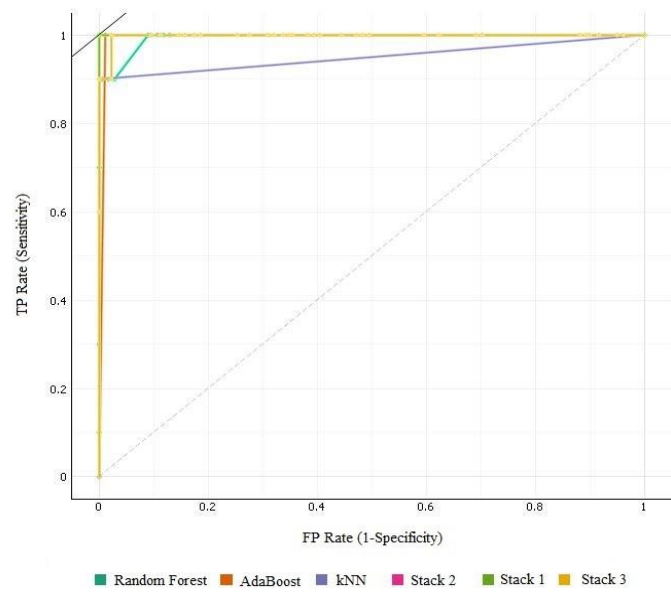

Fig. 4. ROC curve for class Nerium oleander (Inception v3 + Stack 1)
Usually the unique characteristics are manually determined i.e. feature engineering, which is one of the most the most time consuming and expensive process in machine learning. This problem can be overcome by using deep learning. Deep Neural Nets can learn complex functions which are difficult to even comprehend. These networks have activation functions which make full use and derive benefits from non-linear relations. This methodology of reusing pre-trained models for the feature extraction with respect to our task is called as feature learning or representation learning. There are multiple varieties of feature learning. In our paper we have employed Inceptionv3 network [18] for feature learning. The Inception framework was a milestone in the advancement of convolutional neural networks. Before Inception, the popular CNNs simply stacked convolution layers deeper and deeper, expecting to get better performance. It allows the image to have large variations in size. In the previous Inception versions before Inception-v3, it was observed that the auxiliary classifiers, did not had much effect until the very end of the training process, as the accuracies came close to saturation. Inception-v3 overcame this without degrading the performance by using label smoothing, BatchNorm in the auxiliary classifiers, factorized $7 \times 7$ convolutions and RMSProp Optimizer. Even though this model is 42 layers deep, the cost of computation is roughly just twice more than that of GoogLeNet [19], and is significantly more competent than that of VGGNet [16].

Machine learning results can be improved by using Ensemble learning as it combines multiple analytical models. In contrast with a single model, this methodology has significantly better performance in prediction. There are multiple approaches to implement ensemble learning, one being stacking. Multiple regression models or classifications can be combined together by stacking, therefore it applies different models for the same problem as any single model is generally unable to cover the complete space of the problem on its own.

\section{CONCLUSION}

In this study, we have proposed a composite deep learning model which uses a Inception-v3 model for feature mining and stacking model for leaf species classification. Taking 21 different classes of leaves consisting a total of 1287 images, we obtained an accuracy of $99.5 \%$. We have shown that our approach outperformed other contemporary algorithms by the help of various statistical measures. Applications of our work include the convenient classification of plants by their leaf's detection which can be used by anyone to identify any plant and for the better understanding the different types of plants. Farmers can use this to identify an invasive weed or whether a plant's leaves are edible by domesticated animals or not. 


\section{REFERENCES}

1. Vera, A. (1989). Wound healing, oral \& topical activity of Aloe vera. Journal of the American Podiatric Medical Association, 79, 559-562.

2. Gupta, S. K., Prakash, J., \& Srivastava, S. (2002). Validation of traditional claim of Tulsi, Ocimum sanctum Linn. as a medicinal plant.

3. Shahzad, A. (2012). Hemp fiber and its composites-a review. Journal of Composite Materials, 46(8), 973-986.

4. Dev, V. G., Venugopal, J., Sudha, S., Deepika, G., \& Ramakrishna, S. (2009). Dyeing and antimicrobial characteristics of chitosan treated wool fabrics with henna dye. Carbohydrate Polymers, 75(4), 646-650.

5. Jarvill-Taylor, K. J., Anderson, R. A., \& Graves, D. J. (2001). A hydroxychalcone derived from cinnamon functions as a mimetic for insulin in 3T3-L1 adipocytes. Journal of the American College of Nutrition, 20(4), 327336.

6. Rao, P. V., \& Gan, S. H. (2014). Cinnamon: a multifaceted medicinal plant. Evidence-Based Complementary and Alternative Medicine, 2014

7. 周叔平, \& 王新中. (2008). Flower herbal tea used for treatment of menopathies. Journal of Traditional Chinese Medicine, (3), 14.

8. Pearce, J. M. (2008). Leopold Auenbrugger: camphorinduced epilepsy-remedy for manic psychosis. European neurology, 59(1-2), 105-107.

9. Birks, J., \& Evans, J. G. (2009). Ginkgo biloba for cognitive impairment and demen-tia. Cochrane Database of systematic reviews, (1).

10. Yigit, E., Sabanci, K., Toktas, A., \& Kayabasi, A. (2019). A study on visual features of leaves in plant identification using artificial intelligence techniques. Computers and Electron-ics in Agriculture, 156, 369377.

11. Sharma, P., Aggarwal, A., Gupta, A., \& Garg, A. (2019). Leaf Identification Using HOG, KNN, and Neural Networks. In International Conference on Innovative Computing and Communications (pp. 83-91). Springer, Singapore.

12. Aliyu, M. G., Kadir, M. F. A., Mamat, A. R., \& Mohamad, M. (2018). Noise removal using statistical operators for efficient leaf identification. International Journal of Computer Aided Engineering and Technology, 10(4), 364-377.

13. Jalal, A. S., \& Khan, M. (2018). Plant Identification Using Leaf Image Analysis.

14. Du, J. X., Wang, X. F., \& Zhang, G. J. (2007). Leaf shape based plant species recogni-tion. Applied mathematics and computation, 185(2), 883-893.

15. Hossain, J., \& Amin, M. A. (2010, December). Leaf shape identification based plant biomet-rics. In 2010 13th International conference on computer and information technology (ICCIT) (pp. 458-463). IEEE.

16. Wang, Z., Chi, Z., \& Feng, D. (2003). Shape based leaf image retrieval. IEE Proceedings-Vision, Image and Signal Processing, 150(1), 34-43.

17. Rankothge, W. H., Dissanayake, D. M. S. B., Gunathilaka, U. V. K. T., Gunarathna, S. A. C. M., Mudalige, C. M., \& Thilakumara, R. P. (2013, January). Plant recognition system based on Neural Networks. In 2013 International Conference on Advances in Technology and Engineering (ICATE) (pp. 1-4). IEEE.

18. Szegedy, C., Vanhoucke, V., Ioffe, S., Shlens, J., \& Wojna, Z. (2016). Rethinking the in-ception architecture for computer vision. In Proceedings of the IEEE conference on comput-er vision and pattern recognition (pp. 2818-2826).

19. Szegedy, C., Liu, W., Jia, Y., Sermanet, P., Reed, S., Anguelov, D., ... \& Rabinovich, A. (2015). Going deeper with convolutions. In Proceedings of the IEEE conference on comput-er vision and pattern recognition (pp. 1-9).

20. Simonyan, K., \& Zisserman, A. (2014). Very deep convolutional networks for large-scale image recognition. arXiv preprint arXiv:1409.1556.

21. Ben-Hur, A., Horn, D., Siegelmann, H. T., \& Vapnik, V. (2001). Support vector cluster-ing. Journal of machine learning research, 2(Dec), 125-137.

22. Freund, Y., Schapire, R., \& Abe, N. (1999). A short introduction to boosting. Journal-Japanese Society For Artificial Intelligence, 14(771-780), 1612.

23. Lee, Y. (1991). Handwritten digit recognition using $k$ nearest-neighbor, radial-basis func-tion, and backpropagation neural networks. Neural computation, 3(3), 440-449.

24. Bosch, A., Zisserman, A., \& Munoz, X. (2007, October). Image classification using random forests and ferns. In Computer Vision, 2007. ICCV 2007. IEEE 11th International Confer-ence on (pp. 1-8). IEEE.

25. Wu, S. G., Bao, F. S., Xu, E. Y., Wang, Y. X., Chang, Y. F., \& Xiang, Q. L. (2007, De-cember). A leaf recognition algorithm for plant classification using probabilistic neural net-work. In 2007 IEEE international symposium on signal processing and information tech-nology $(\mathrm{pp}$ 11-16). IEEE. 